\title{
VLBI AND VLA OBSERVATIONS OF SCO X-1
}

\author{
B. J. Geldzahler \\ Applied Research Corporation \\ Landover, MD \\ E. B. Fomalont \\ National Radio Astronomy Observatory \\ Charlottesville, VA 22903 \\ N. L. Cohen
Boston University
Boston, MA 02215
}

We have measured the absolute and relative positions of the components of Sco X-1 (Fig. 1) with the VLA from 1980-1986 (Geldzahler, Fomalont, Hilldrup, and Corey, 1981, A.J., 86, 1036; Fomalont, Geldzahler, Hjellming, and Wade, 1983, Ap.J., 275, 802; Geldzahler and Fomalont, 1986, Ap.J., 313, 815). We have used these observations to test the dual-beam model of radio sources proposed by Blandford and Rees (1974). Our latest VLA results may be summarized as follows:

1. All radio components of Sco $\mathrm{X}-1$ have an absolute proper motion that is nonzero and consistent with the proper motion of the optical star. Thus, there is no doubt of the physical association of the components with the x-ray emitting star.

2. The NE lobe is moving radially away from the core at a rate of $0.015 \pm 0.002$ $\operatorname{arcsec} \mathrm{yr}^{-1}$, corresponding to a transverse velocity of $35 \pm 5 \mathrm{~km} \mathrm{~s}^{-1}$ at an assumed distance of 500 parsecs.

3. The relative proper motion of the core and the SW hot spot at the outer edge of the SW lobe is $<0.030$ arcsec $\mathrm{yr}^{-1}$.

4. The assumption of linear motion of all three components is consistent with the post-fit residuals.

5. The flux density of the core can vary by an order of magnitude in a period of an hour.

6. The NE lobe varies by about $10 \%$ over periods of a year or less.

7. The SW hot spot does not appear to vary within the errors. However, because it is faint, percentage variations as large as those of the NE lobe could be present and may be reflected in the actual flux density values of the hot spot.

8. The integrated flux density of the SW lobe varies by about $30 \%$ over time scales of less than one year, and variations are not localized to a small region in the lobe.

9. There may be a correlation between the flux density variation in the NE lobe, SW hot spot, and SW lobe. 
With $18 \mathrm{~cm}$ VLBI observations of the NE lobe in 1985, we measured a lobe size of $\sim 7$ mas, but the source is weak and the consequent noisiness of the data allows for $\sim 20 \%$ halo. Our first VLBI study used NRL, HRAS, VLA (phased array), and OVRO.

Our second epoch $18 \mathrm{~cm}$ VLBI observations of Sco X-1 used Green Bank, HRAS, VLA (phased array, D configuration), and OVRO. We positioned the VLA phase center midway between the NE lobe and the core such that both sources were in the beam. The results of this study are: (1) the confirmation of the amazingly small size of the NE lobe, and (2) the measurement of the offset of the NE lobe and the core to milliarcsec accuracy. Thus, we have reduced the measurement errors by an order of magnitude over the VLA results. The measurement of the offset was achieved because we were successful in detecting the core, at a $S / N \sim 30$, through phase referencing of the core to the NE lobe. Subsequent VLBI observations of the relative offsets should be sensitive enough to look for nonlinear motions.

There are several problems that remain in understanding the radio results of Sco X-1. The measured size of the NE lobe, $<7$ mas, is an upper limit so the true size of the component is unknown. Confinement of this lobe seems to require an exceptionally large magnetic field or dense ambient medium under conventional emission processes. The uncertainty of the distance to Sco X-1 causes uncertainties in the application of the dual-beam model. We plan to measure the distance with the Hubble Space Telescope.

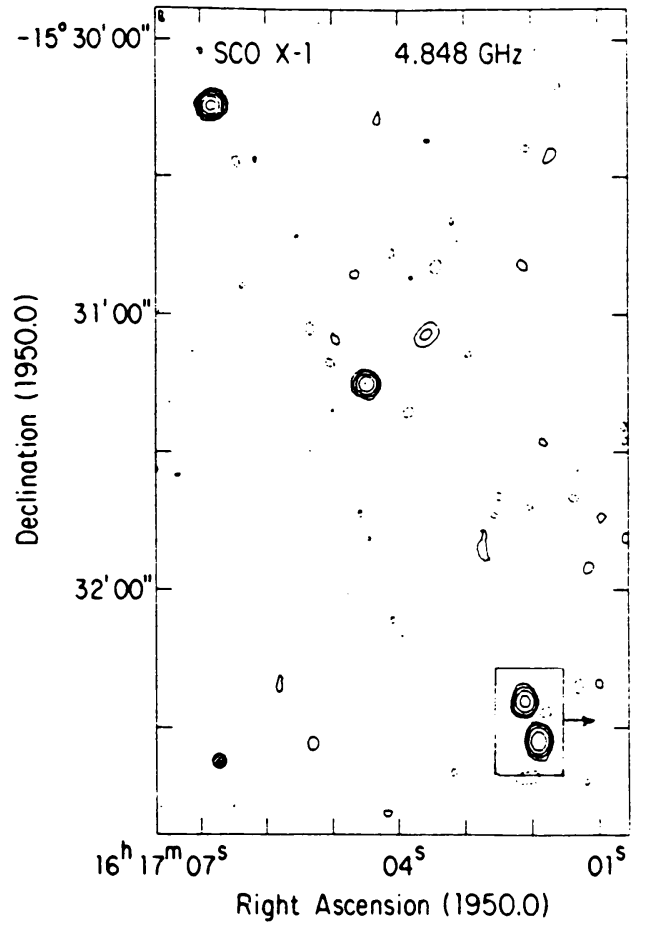

Figure 1

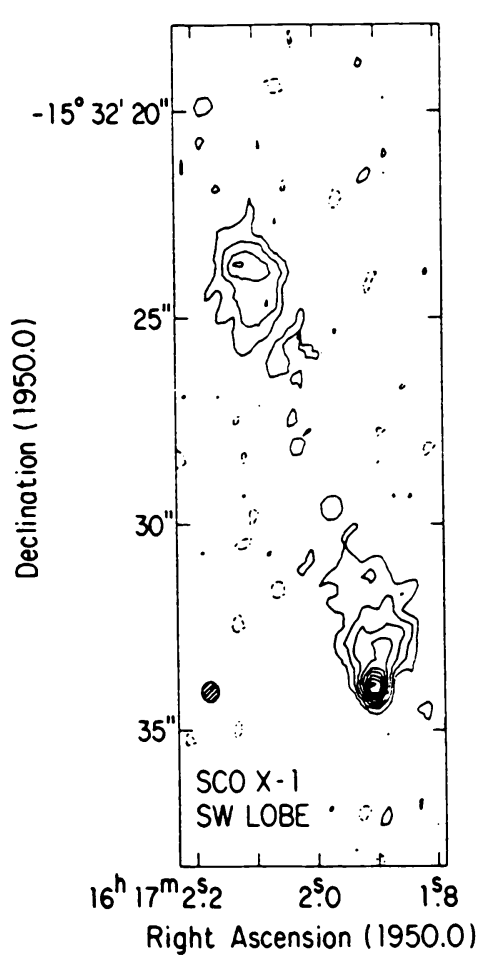

Right Ascension (1950.0) 